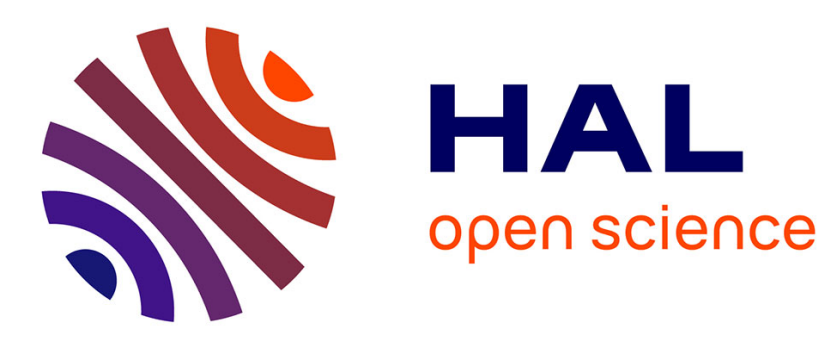

\title{
Using elementary Reactions to Model Growth Processes of Polyaromatic Hydrocarbons under Pyrolysis Conditions of Light Feedstocks
}

Karen Hemelsoet, Veronique van Speybroeck, Kevin M. van Geem, Guy B Marin, Michel Waroquier

\section{To cite this version:}

Karen Hemelsoet, Veronique van Speybroeck, Kevin M. van Geem, Guy B Marin, Michel Waroquier. Using elementary Reactions to Model Growth Processes of Polyaromatic Hydrocarbons under Pyrolysis Conditions of Light Feedstocks. Molecular Simulation, 2008, 34 (02), pp.193-199. 10.1080/08927020801930588 . hal-00515027

\section{HAL Id: hal-00515027 \\ https://hal.science/hal-00515027}

Submitted on 4 Sep 2010

HAL is a multi-disciplinary open access archive for the deposit and dissemination of scientific research documents, whether they are published or not. The documents may come from teaching and research institutions in France or abroad, or from public or private research centers.
L'archive ouverte pluridisciplinaire HAL, est destinée au dépôt et à la diffusion de documents scientifiques de niveau recherche, publiés ou non, émanant des établissements d'enseignement et de recherche français ou étrangers, des laboratoires publics ou privés. 


\section{Molecular Simulation}

Journal of

Experimental Nanoscience

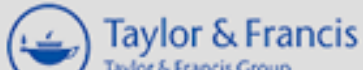

Taylor \& Francis Group

\section{Using elementary Reactions to Model Growth Processes of Polyaromatic Hydrocarbons under Pyrolysis Conditions of Light Feedstocks}

\begin{tabular}{|r|l|}
\hline Journal: & Molecular Simulation/Journal of Experimental Nanoscience \\
\hline Manuscript ID: & GMOS-2007-0171.R1 \\
\hline Journal: & Molecular Simulation \\
\hline Date Submitted by the \\
Author: & 09-Jan-2008 \\
\hline Complete List of Authors: & $\begin{array}{l}\text { Hemelsoet, Karen; Ghent University, Center for Molecular Modeling } \\
\text { Van Speybroeck, Veronique; Ghent University, Center for molecular } \\
\text { modeling } \\
\text { Van Geem, Kevin; Ghent University, Laboratory for Chemical } \\
\text { Technology } \\
\text { Marin, Guy; Ghent University, Laboratory for Chemical Technology } \\
\text { Waroquier, Michel; Ghent University, Center for Molecular Modeling }\end{array}$ \\
\hline Keywords: & $\begin{array}{l}\text { DFT calculations, polyaromatic hydrocarbons, bond dissociation } \\
\text { enthalpy, carbon-centered radicals, hydrogen abstraction }\end{array}$ \\
\hline
\end{tabular}

\section{SCHOLARONE" Manuscripts}


(PLEASE LEAVE THESE VOLUME/ISSUE DETAILS TO BE ASSIGNED BY JOURNALS PRODUCTION AT A LATER STAGE)
Running heads
(verso)
K. Hemelsoet et al.
(DO NOT INCLUDE THIS AT FIRST SUBMISSION FOR BLIND REVIEW, BUT DO INCLUDE IT WHEN PREPARING THE FINALLY ACCEPTED MANUSCRIPT FOR SUBMISSION)
(recto) Molecular Simulation

\title{
Using elementary Reactions to Model Growth Processes of Polyaromatic Hydrocarbons under Pyrolysis Conditions of Light Feedstocks
}

\author{
K. Hemelsoet, ${ }^{\mathrm{a}}$ V. Van Speybroeck, ${ }^{\mathrm{a}^{*}}$ K. M. Van Geem, ${ }^{\mathrm{b}}$ G. B. Marin ${ }^{\mathrm{b}}$ and M. Waroquier ${ }^{\mathrm{a}}$ \\ ${ }^{\mathrm{a}}$ Center for Molecular Modeling, Ghent University, Proeftuinstraat 86, 9000 Ghent, Belgium; ${ }^{\mathrm{b}}$ Laboratory for Chemical \\ Technology, Ghent University, Krijgslaan 281-S5, 9000 Ghent, Belgium
}

\begin{abstract}
Density Functional Theory results are presented for elementary steps leading to coke growth within a steam cracking unit. The discussed pathway starts from toluene and ultimately, 1-methylnaphthalene is formed. In order to find the rate determining step for coke formation, the pseudo first-order rate coefficients of the various steps are compared taking into account the concentrations of diverse coke precursors. The influence of the polyaromatic environment is studied for a large set of methylated polycyclic aromatic molecules, by means of carbon-hydrogen bond dissociation enthalpy values. Subsequent hydrogen abstraction reactions at the methylated polyaromatics, by a methyl radical, are also examined. The abstraction is found to preferentially occur at the larger systems and is in general faster compared to abstractions at the analogous non-methylated species.
\end{abstract}

Keywords: DFT calculations, polyaromatic hydrocarbons, bond dissociation enthalpy, carbon-centered radicals, hydrogen abstraction 
*Corresponding author:

Prof. Dr. ir. Veronique Van Speybroeck

Center for Molecular Modeling, Ghent University

Proeftuinstraat 86, 9000 Ghent, Belgium

Tel: +329264 6558 Fax:+3292646697

Email: Veronique.Vanspeybroeck@ugent.be

Other authors:

Dr. ir. Karen Hemelsoet

Email: Karen.Hemelsoet@ugent.be

Dr. ir. Kevin M. Van Geem

Email: Kevin.Vangeem@ugent.be

Prof. Dr. ir. Guy B. Marin

Email: Guy.Marin@ugent.be

Prof. Dr. Michel Waroquier

Email: Michel.Waroquier@ugent.be

Word count: The text-document consist of a total of 4226 words, including all titles, figure captions, tables and references.

The paper is for a special issue, organised by Prof. Delhommelle, following the AIChE07 Annual Meeting conference. 
(PLEASE LEAVE THESE VOLUME/ISSUE DETAILS TO BE ASSIGNED BY JOURNALS PRODUCTION AT A LATER STAGE)

\title{
Using elementary Reactions to Model Growth Processes of Polyaromatic Hydrocarbons under Pyrolysis Conditions of Light Feedstocks
}

\begin{abstract}
Density Functional Theory results are presented for elementary steps leading to coke growth within a steam cracking unit. The discussed pathway starts from toluene and ultimately, 1-methylnaphthalene is formed. In order to find the rate determining step for coke formation, the pseudo first-order rate coefficients of the various steps are compared taking into account the concentrations of diverse coke precursors. The influence of the polyaromatic environment is studied for a large set of methylated polycyclic aromatic molecules, by means of carbon-hydrogen bond dissociation enthalpy values. Subsequent hydrogen abstraction reactions at the methylated polyaromatics, by a methyl radical, are also examined. The abstraction is found to preferentially occur at the larger systems and is in general faster compared to abstractions at the analogous non-methylated species.
\end{abstract}

Keywords: DFT calculations, polyaromatic hydrocarbons, carbon-centered radicals, hydrogen abstraction, coke formation

\section{Introduction}

Various free radical mechanisms leading to the growth of polyaromatic species have in recent years intensively been examined using ab initio modelling techniques [1-5]. In this light, the presenting authors have provided important contributions focussing on coke growth within a steam cracking unit [6-11]. Steam cracking of large hydrocarbons remains at present the most important production source of light olefins such as ethylene and propylene. Simultaneously with the cracking reactions, the growth of a coke layer at the inner wall of the reactor tube is taking place. The presence of this layer reduces the efficiency of the cracking unit substantially and its growth is thus highly undesirable. In practice, thousands of reactions are taking place at the same time, prohibiting an extended experimental or computational investigation. In order to obtain more insight into this complex mechanism, models consisting of various elementary steps have been proposed [12, 13]. Five classes of reversible reactions have been presented, including hydrogen abstraction, addition, substitution and cyclization. The main concern is to establish the rate determining step for coke formation. In order to do so, ab initio modelling techniques are used to compute the energies of the involved molecules and 
the kinetics of the corresponding radical reactions. Additional information about the concentrations of the coke precursors is obtained using single-event micro-kinetic simulation techniques, which in particular allows a comparison between the pseudo-first order (or modified) rate coefficients of the various elementary reactions [14]. Due to its reactive characteristics, the coke layer can be modelled as a network of aromatic rings. Initially, the coke growth process is modelled by investigating the route from benzene to naphthalene or naphthalene-like species [11]. In a next step, the influence of the polyaromatic environment is examined using an extended set of polycyclic aromatic hydrocarbons (PAHs) as model representatives [10]. Knowledge of the thermodynamic quantities of the latter species remains in general an ongoing challenge due to their high importance in a large variety of fields (such as incomplete combustion processes, astrophysics, fullerene chemistry ...) $[15,16]$. In addition, PAHs also form the largest class of carcinogenic and mutagenic species $[17,18]$.

Both thermodynamic and kinetic properties have been calculated using ab initio techniques. As the involved species are considerably large, density functional theory is preferred over post-Hartree-Fock methods due to the interesting accuracy-computational cost ratio of the former theory. At present, the performance of the newest class of DFT-functionals is known to be of sufficiently high quality leading to accurate and reliable data [19]. This aspect has in particular been shown for the essential class of hydrogen abstractions leading to arylic radicals by the presenting authors $[20,10]$.

In the present contribution, various topics are being discussed. Firstly, DFT-results corresponding to elementary reactions for a pathway starting from toluene and leading to 1-methylnaphthalene are given. So far, computational studies on methylated polyaromatics have been very limited [21]. The latter species are nevertheless known to play a key role within combustion methods. Abstraction of a methyl hydrogen leads to the class of benzylic radicals, in which the stability is enhanced due to a well-known resonance effect [22]. The influence of the polyaromatic environment is thus expected to be substantially larger compared to the series of arylic radicals. Secondly, the importance of benzylic radicals in growth processes of polyaromatics is highlighted further. An extended amount of bond dissociation enthalpies (BDEs) is computed in order to assess the reactivity of the species. Thirdly, radical hydrogen abstractions at a series of methylated polyaromatics using an assisting methyl radical are investigated.

\section{Methodology}

All calculations were performed using the Gaussian03 software package [23]. The optimization of geometries as well as frequency computations were performed using the B3LYP functional [24, 25], in combination with a basis set of moderate size $(6-31 G+G(d, p)$ or $6-311 G(d, p))$. Based on other studies involving similar radical reactions, this method has proven to provide a reliable and quantitatively good description. For the computation of the thermodynamic and kinetic parameters of the studied hydrogen abstraction reactions, a factor of $0.9806,0.9614$ and 0.9989 was used to scale the zero-point vibrational energies (ZPVEs), the harmonic frequencies and the thermal correction to the enthalpy, respectively. The use of scale factors provides a means for accounting for systematic deviations between measured and computed frequencydependent properties, and is an important consideration for an accurate ab initio description [26]. Subsequent single-point energy computations were carried out applying the hybrid meta BMK functional [27], in combination with the extended $6-311+\mathrm{G}(3 \mathrm{df}, 2 \mathrm{p})$ basis set. This particular functional was earlier shown to be accurate within approximately $10 \mathrm{~kJ} / \mathrm{mol}$ for the calculation of reaction barriers [27]. Moreover, it provides precise thermodynamic and kinetic results for a variety of similar species and hydrogen abstraction reactions 
at a feasible computational cost $[19,20,28]$. We applied traditional transition state theory (TST) to obtain intrinsic rate coefficients for the hydrogen abstraction reactions at methylated PAHs. Special attention was given to the accurate treatment of internal rotations of the methyl tops, present in both the reactants and the transition structures [29]. The accuracy of the results was increased by incorporating Eckart tunneling corrections [30].

\section{Results and Discussion}

\subsection{Network starting from toluene}

Compared to our earlier work on pure PAHs, we now start from a methylated species (toluene). The latter molecule is created following a substitution reaction at benzene (Scheme 1): an initial addition reaction of a methyl radical at benzene is followed by a hydrogen abstraction reaction. We earlier reported that this initial addition reaction of $\mathrm{CH}_{3}$ is highly competitive with the hydrogen abstraction reaction [11]. Toluene-like species can also be formed within the coke surface following other reactions, e.g. the addition of propene, and will inherently be present in the network.

Scheme 1: Substitution reaction starting from benzene and leading to toluene.

A reaction route, starting from toluene (1-1) and leading to 1-methylnaphthalene (2-1) is presented in Scheme 2 (for the labeling of the species, see Figure 3). Analogous to earlier work, the pathway consists of consecutive elementary steps. The initial hydrogen abstraction reaction $(R l)$ is followed by two additions of ethylene $(R 2$ and $R 3)$. Two successive isomerisation reactions ( $R 4$ and $R 5)$ are observed, and are followed by a cyclization reaction $(R \sigma)$. In order to restore the aromaticity of the newly formed ring, five dehydrogenation reactions are needed ( $R 7$ to $R 11)$. Ultimately, 1-methylnaphthalene is formed.

| Scheme 2: Reaction pathway starting from toluene (1-1) and leading to 1-methylnaphthalene (2-1) (labeling see Figure 3).

It is important to highlight that knowledge of the intrinsic rate coefficients $k(\mathrm{~T})$ is not sufficient in order to determine the rate limiting step of the entire pathway which contains both bimolecular and unimolecular reactions. The concentrations of the coke precursors have been taken into account. Pseudo first-order (or modified) rate coefficients $k^{\prime}(\mathrm{T})$ which are expressed in units $1 / \mathrm{s}$ are used:

$r=k(T) c_{A} c_{B}=k^{\prime}(T) c_{A}$

The concentrations of the methyl radical, the ethylene molecule and the hydrogen radical were obtained from a 2-dimensional reactor model developed at the Laboratory for Chemical Technology [14]. The radical coke precursors are present in much lower concentrations compared to the olefinic species in the gas phase and this has a huge effect on the relative ratio of the modified rate coefficients. This is illustrated in Figure 1, where the intrinsic and modified rate coefficients are given for the initial addition reaction of a methyl radical at benzene (see Scheme 1). In the left parts of (a) and (b), the values obtained for the entire reactor tube are given. The $\mathrm{x}$-axis corresponds to the relative radial coordinate with $\mathrm{x}=0.0$ corresponding to the central axis 
and $x=1.0$ to the wall of the tube. The $y$-axis corresponds to the axial coordinate. In the right parts of (a) and (b), the part close to the wall of the tube where coke formation is initialised for is given in more detail. The influence of the concentration of the methyl radical is enormous; the rate coefficient calculated at the tube wall for the reaction under consideration is reduced $\left(k^{\prime}(\mathrm{T})\right.$ compared to $\left.k(\mathrm{~T})\right)$ with approximately a factor of 10000 .

Figure 1: Intrinsic (a) and modified (b) rate coefficients for the addition reaction of a methyl radical to benzene (see Scheme 1).

We now return to the reaction network depicted in Figure 1. An overview of the pseudo first-order rate coefficients $k^{\prime}(\mathrm{T})$ (in 1/s) obtained near the wall of the reactor tube outlet is given in Figure 2 . In analogy with earlier results (the reaction network from benzene to naphthalene [11]), the initial hydrogen abstraction at toluene $(R I)$ and the final dehydrogenation reactions (going from a neutral to a radical species: $R 8$ and $R 10$ ) are found to be the crucial steps, as their respective $k^{\prime}(\mathrm{T})$ values are the smallest.

Figure 2: Modified rate coefficients $k^{\prime}(\mathrm{T})$ for the forward (in light grey) and reverse (in dark grey) reaction steps of the entire network (as defined in Scheme 2).

\subsection{BDEs of benzylic radicals}

In previous paragraph, we demonstrated the importance of toluene and the subsequently created benzyl radical as a starting point for the coke growth model. Moreover, we pointed out the importance of benzylic radicals for general growth processes of polyaromatics. In a next step an extended set of singly methylated species, as depicted in Figure 3 is studied to account for the influence of the polyaromatic environment. The numbering of the various methylated sites is conform the IUPAC convention.

More precisely, carbon-hydrogen bond dissociation enthalpies (BDEs), corresponding to the bond breaking of a methyl hydrogen, are calculated using the reliable and yet cost-effective BMK/6-311+G(3df,2p)//B3LYP/6$31+\mathrm{G}(\mathrm{d}, \mathrm{p})$ level of theory. Izgorodina et al. reported on the potential problems using DFT functionals for reproducing reliable trends in BDE values as well as on the very reasonable performance of the BMK functional for the computation of a large set of absolute BDEs [31]. The results are given in Table 1. First of all we note that the BDE values of benzylic radicals are relatively small. This is in accordance with the enhanced stability of these species due to well-known resonance effects.

Figure 3: Singly methylated PAHs, methylation can occur at various positions as indicated (the methyl group is not displayed in the | Figure).

For the subcategory of non-linear species the effect of the polyaromatic environment is found to be smaller than expected. E. g. the BDE value of the large methylated coronene molecule (structure 13) deviates in a minor way (i.e. with $1.26 \mathrm{~kJ} / \mathrm{mol}$ ) from the reference BDE value of toluene (structure 1). It is overall concluded that an enlarged polyaromatic environment (leading to an enhanced delocalization possibility) does not lead to a substantially smaller BDE value. The behaviour of the series of linear methylated PAHs (structures $\mathbf{1}$ to $\mathbf{5}$ ) is nevertheless very peculiar. Two distinct and equally important features can be 
distinguished. First of all, the BDE values do decrease with increasing molecular size for this important subcategory. The calculated BDE value of $301 \mathrm{~kJ} / \mathrm{mol}$ for 5-13 deviates strongly from the BDE value at toluene $(1-1,373 \mathrm{~kJ} / \mathrm{mol})$, being the smallest representative of the linear series. Secondly, the BDE values strongly depend on the site of methylation, as is in particular seen for the structures $\mathbf{3}, \mathbf{4}$ and $\mathbf{5}$. This must be traced back to characteristic geometrical features - and in particular closely positioned hydrogen atoms introducing strong repulsive effects - in the optimized methylated reactant structures.

\subsection{Hydrogen abstraction reactions at methylated PAHs}

In a next step, abstraction reactions of methyl hydrogen at the proposed set of methylated PAHs (Figure 3) using an assisting methyl radical are considered. In this way, a benzylic radical and methane are formed. Thermodynamic and kinetic properties are computed, using BMK/6-311+G(3df,2p) energies and B3LYP/6$31+\mathrm{G}(\mathrm{d}, \mathrm{p})$ geometries. For the computation of intrinsic rate coefficients, Eckart tunneling corrections are included in order to improve the accuracy of the standard TST results. It was however impossible to identify the majority of the internal rotations of the methyl tops (present in both the reactants and the transition states) as independent movements within the vibrational spectrum and therefore, all motions were treated using the harmonic oscillator $(\mathrm{HO})$ model.

First of all it is noted that the geometrical features of the transition states indicate that the forming bond is always longer than the breaking bond, a behaviour which is in correspondence with the enhanced stability of the product radicals: the transition states can be described as "early" in the Hammond sense, as they geometrically resemble the reactants. Opposite to the series of pure (or non-methylated) PAHs, the influence of the planarity of the aromatic substrate is less substantial. Moreover, in case of the transition structures the methylation usually results in a non-planar structure in which the attached methyl group is twisted away from the aromatic substrate. Consequently, the attacking gas phase radical (in this particular case a methyl radical) encounters relatively little or no steric hindrance when approaching the site of abstraction.

Secondly, the thermodynamic properties are calculated using scaled ZPVEs and thermal corrections to the enthalpy. The reaction barriers at 0 Kelvin $\left(\Delta \mathrm{E}_{0}\right)$ and the reaction enthalpies at $298 \mathrm{~K}\left(\Delta \mathrm{H}_{298}\right)$ reveal interesting characteristics. The latter values are of course closely related to the BDE results which were discussed in section 3.2. For the present contribution, it is important to address the reactivity-enthalpy relationship. We earlier found that for the series of abstraction at non-methylated PAHs (leading to the formation of arylic radicals), a normal reactivity-enthalpy relationship could only be found for hydrogen abstractions at the subcategory of linear acenes as in these cases, steric hindrance effects between the aromatic substrate on one hand and the incoming methyl radical on the other, are non-existent [10]. For the present species we earlier mentioned that steric effects are not important for the majority of the transition structures. And indeed, a normal reactivity-enthalpy was overall obtained: a large stabilization effect in the product radical (large absolute $\Delta \mathrm{H}_{298}$ value) corresponds to an enhanced reactivity (small $\Delta \mathrm{E}_{0}$ value). This behaviour is depicted in Figure 4. Several exceptional points can however be discerned within the Figure. They correspond to reactions with barriers around (or larger than) $50 \mathrm{~kJ} / \mathrm{mol}$ and they mostly correspond to strongly congested sites (such as 11-16 and 12-14).

Figure 4: Reactivity-enthalpy relationship for the hydrogen abstraction reactions at methylated PAHs by an attacking methyl radical. 
Thirdly, the rate coefficients $k(\mathrm{~T})$ and derived kinetic properties (activation energy $\mathrm{E}_{\mathrm{a}}$ and pre-exponential factor A) are computed for the temperature interval between 700 and $1100 \mathrm{~K}$, which is relevant for the coke growth process. It is noted that, compared to abstractions at pure PAHs, the majority of the abstractions at methylated PAHs occurs faster. This is illustrated in Figure 5, for a random selection of five reactions. Beside the abstraction at the reference molecule (benzene and toluene, respectively), 2 abstractions at linear species (in particular 3-9 and 5-2) and 2 abstractions at non-linear species (10-14 and 11-1) are selected. It is indeed seen that for the majority of the cases, the abstraction at methylated species are faster in comparison with abstractions at non-methylated PAHs. Although it is hard to draw conclusions for the entire test set, it is in general found that abstraction is easier at larger systems. These results underline the importance of methylated species and the corresponding hydrogen abstraction reactions to initiate radical sites within a polyaromatic surface.

Figure 5: Rate constants $\ln k(\mathrm{~T})\left(\mathrm{k}(\mathrm{T})\right.$ in $\mathrm{m}^{3} / \mathrm{mol} / \mathrm{s}$ ) as a function of 1/T (in 1/K) for a random selection of five hydrogen abstraction reactions (numbering is conform Figure 3). The full lines correspond to abstractions at pure PAHs (leading to arylic radicals) whereas the dotted lines correspond to abstractions at methylated PAHs (leading to benzylic radicals).

\section{Conclusions}

In this contribution we provided an overview of recent results obtained within the ab initio study of radical reactions leading to the growth of polyaromatic species. The examined growth process can be situated within the industrially important application of coke formation at the inner wall of a reactor tube used in a steam cracking procedure. The latter remains at present a central production methodology of light olefins such as ethylene and propylene.

First of all, a new reaction route starting from toluene and leading to 1-methylnaphthalene has been proposed. A comparison between the pseudo first-order rate coefficients of all elementary reactions indicates that the initial hydrogen abstraction reaction at toluene (leading to the formation of the benzyl radical) and the final dehydrogenation reactions are the rate limiting steps. The importance of benzylic radicals was also highlighted. Consequently, carbon-hydrogen bond dissociation energies (BDEs) were calculated for an extended set of methylated PAHs, being model representatives for the coke surface. The effect of the polyaromatic environment on BDEs is found to be smaller than expected for the subcategory of non-linear species. The subcategory of linear methylated PAHs on the other hand reveals peculiar behaviour. First of all, the BDE values decrease with increasing molecular size. And secondly, the BDE values depend in a very substantial way on the site of methylation. Finally, hydrogen abstraction reactions at the set of methylated PAHs using an assisting methyl radical were closely investigated. The abstraction is found to preferentially occur at the larger systems and is in general faster compared to abstractions at the analogous non-methylated species. In this contribution, the presented overview of reliable DFT-results on methylated species and derived benzylic radicals underlines their importance within general growth schemes of polyaromatic structures. The corresponding hydrogen abstraction reactions are found to be key reactions to initiate radical sites within the polyaromatic surface.

\section{Acknowledgements}

This work is supported by the Fund for Scientific Research-Flanders (FWO) and the Research Board of Ghent University. 


\section{References}

[1] L. Vereecken, J. Peeters, H. F. Bettinger, R. I. Kaiser, P. v. R. Schleyer, H. F. Schaefer, III, Reaction of phenyl radicals with propyne, J. Am. Chem. Soc. 124 (2002), 2781.

[2] T. J. M. de Bruin, F. Lorant, H. Toulhoat, W. A. Goddard, III, Reaction kinetics of a selected number of elementary processes involved in the thermal decomposition of 9-methylphenanthrene using density functional theory, J. Phys. Chem. A 108 (2004), 10302.

[3] J. Appel, H. Bockhorn, M. Frenklach, Kinetic modelling of soot formation with detailed chemistry and physics: laminar premixed flames of C-2 hydrocarbons, Comb. And Flame 121 (2000), 122.

[4] V. V. Kislov, A. M. Mebel, The formation of naphthalene, azulene, and fulvalene from cyclic C-5 species in combustion: an ab initio/RRKM study of 9-h-fulvalenyl (C5H5-C5H4) radical rearrangements, J. Phys. Chem. A 111 (2007), 9532.

[5] C. Cavallotti, S. Mancarella, R. Rota, S. Carra, Conversion of C5 into C6 cyclic species through the formation of C7 intermediates, J. Phys. Chem. A 111 (2007), 3959.

[6] V. Van Speybroeck, M. F. Reyniers, G. B. Marin, M. Waroquier, The kinetics of cyclization reactions on polyaromatic from first principles, ChemPhysChem 3 (2002), 863.

[7] M. Saeys, M. F. Reyniers, G. B. Marin, V. Van Speybroeck, M. Waroquier, Ab initio group contribution method for activation energies for radical additions, AIChE J. 50 (2004), 426.

[8] M. Saeys, M. F. Reyniers, V. Van Speybroeck, M. Waroquier, G. B. Marin, Ab initio group contribution method for activation energies of hydrogen abstraction reactions, ChemPhysChem 7 (2006), 188.

[9] V. Van Speybroeck, G. B. Marin, M. Waroquier, Hydrocarbon bond dissocation enthalpies: from substituted aromatics to polyaromatics, ChemPhysChem 7 (2006), 2205.

[10] K. Hemelsoet, V. Van Speybroeck, D. Moran, G. B. Marin, L. Radom, M. Waroquier, Thermochemistry and kinetics of hydrogen abstraction by methyl radical from polycyclic aromatic hydrocarbons, J. Phys. Chem. A 110 (2006), 13624.

[11] V. Van Speybroeck, K. Hemelsoet, B. Minner, G. B. Marin, M. Waroquier, Modeling elementary reactions in coke formation from first principles, Mol. Sim. 33 (2007), 879.

[12] S. Wauters, G. B. Marin, Kinetic modeling of coke formation during steam cracking, Ind. Eng. Chem. Res. 41 (2002), 2379.

[13] S. Wauters, G. B. Marin, Computer generation of a network of elementary steps for coke formation during the thermal cracking of hydrocarbons, Chem. Eng. J. 82 (2001), 267.

[14] K. M. Van Geem, G. J. Heynderickx and G. B. Marin G. B, Effect of radial temperature profiles on yields in steam cracking, AIChE J. 50 (2004), 173.

[15] L. J. Allamandola, Benzenoid hydrocarbons in space - the evidence and implications, Top. Curr. Chem. 153 (1990), 1.

[16] A. Léger, L. d'Hendecourt, N. Boccara, Polycyclic Aromatic Hydrocarbons and Astrophysics; Eds.; NATO ASI Series C; Reidel: Dordrecht; The Netherlands 1987, Vol. 191.

[17] R. G. Harvey, Polycyclic Aromatic Hydrocarbons: Chemistry and Carcinogenicity; Cambridge University Press: Cambridge, U. K. 1991.

[18] M. F. Denissenko, A. Pao, M. S. Tang, G. P. Pfeifer, Preferential formation of benzo[a]pyrene adducts at lung cancer mutational hotspots in P53, Science 274 (1996), 430.

[19] M. L. Coote, Reliable theoretical procedures for the calculation of electronic-structure information in hydrogen abstraction reactions, J. Phys. Chem. A 108 (2004), 3865.

[20] K. Hemelsoet, D. Moran, V. Van Speybroeck, M. Waroquier, L. Radom, An assessment of theoretical procedures for predicting the thermochemistry and kinetics of hydrogen abstraction by methyl radical from benzene, $\mathrm{J}$. Phys. Chem. A 110 (2006), 8942.

[21] J. P. Leininger, C. Minot, F. Lorant, F. Behar, Density functional theory investigation of competitive free-radical processes during the thermal cracking of methylated polyaromatics: estimation of kinetic parameters, J. Phys. Chem. A 111 (2007), 3082.

[22] D. A. Hrovat, W. T. Borden, Ab-initio calculations of the relative resonance stabilization energies of allyl and benzyl radicals, J. Phys. Chem. 98 (1994), 10460. 
[23] Frisch, M. J.; et al., GAUSSIAN03, Revision C.02, Gaussian, Inc., Wallingford, CT, 2004.

[24] A. D. Becke, Density-functional thermochemistry 3. The role of exact exchange, J. Chem. Phys. 98 (1993), 5648.

[25] C. Lee, W. Yang, R.G. Parr, Development of the Colle-Salvetti correlation-energy formula into a functional of the electron-density, Phys. Rev. B 37 (1988), 785.

[26] A. P. Scott, L. Radom, Harmonic vibrational frequencies: an evaluation of Hartree-Fock, Moller-Plesset, quadratic configuration interaction, density functional theory, and semiempirical scale factors, J. Phys. Chem. 100 (1996), 16502.

[27] A. D. Boese, J. M. L. Martin, Development of density functionals for thermochemical kinetics, J. Chem. Phys. 121 (2004), 3405.

[28] A. G. Vandeputte, M. K. Sabbe, M.-F. Reyniers, V. Van Speybroeck, M. Waroquier, G. B. Marin, Theoretical Study of the Thermodynamics and Kinetics of Hydrogen Abstractions from Hydrocarbons, J. Phys. Chem. A 111 (2007) 11771.

[29] (a) V. Van Speybroeck, D. Van Neck, M. Waroquier, S. Wauters, M. Saeys, G. B. Marin, Ab initio study on elementary radical reactions in coke formation, Int. J. Quantum Chem. 91 (2003), 384. (b) P. Vansteenkiste, V. Van Speybroeck, G. B. Marin, M. Waroquier, Ab initio calculation of entropy and heat capacity of gas-pahse nalkanes using internal rotations, J. Phys. Chem. A 107 (2003), 3139. (c) P. Vansteenkiste, D. Van Neck, V. Van Speybroeck and M. Waroquier, An extended hindered-rotor model with incorporation of Coriolis and vibrational-rotational coupling for calculating partition functions and derived quantities, J. Chem. Phys. 125 (2006), 049902.

[30] C. Eckart, The penetration of a potential barrier by electrons, Phys. Rev. 35 (1930), 1303

[31] E. I. Izgorodina, M. L. Coote, L. Radom, Trends in $R$-X Bond Dissociation Energies $(R=M e, E t, i-P r, t-B u ; X=H$, $\left.\mathrm{CH}_{3}, \mathrm{OCH}_{3}, \mathrm{OH}, \mathrm{F}\right)$ : A Surprising Shortcoming of Density Functional Theory, J. Phys. Chem. A 109 (2005), 7558. 
Table 1: BDE values of set of methylated PAHs. $B D E_{298}(R-H)=\left[\Delta_{f} H_{298}(R)+\Delta_{f} H_{298}(H)\right]-\Delta_{f} H_{298}(R-H)$ with $\Delta_{f} H_{298}$ the enthalpies of formation of the radical species $(\mathrm{R})$ and molecule $(\mathrm{R}-\mathrm{H})$, respectively. The following nomenclature (referring to Figure 3) is used: the bold number corresponds to the compound, whereas the second number refers to the isomeric position.

\begin{tabular}{|c|c|c|c|c|c|c|c|c|c|c|c|}
\hline PAH & BDE & PAH & BDE & PAH & BDE & PAH & BDE & PAH & BDE & PAH & BDE \\
\hline $1-1$ & 372.83 & $5-13$ & 301.29 & $7-1$ & 365.12 & $8-7$ & 369.94 & 10-12 & 373.67 & \begin{tabular}{|l|}
$14-2$ \\
\end{tabular} & 374.24 \\
\hline $2-1$ & 368.53 & 5-14 & 315.11 & $7-2$ & 369.00 & 8-1 & 367.36 & 11-1 & 366.00 & 14-10 & 368.70 \\
\hline $2-2$ & 370.00 & $5-1$ & 355.63 & $7-3$ & 370.77 & 8-2 & 370.71 & 11-16 & 341.51 & 15-1 & 351.50 \\
\hline 3-9 & 345.83 & $5-2$ & 351.56 & $7-4$ & 370.33 & $8-3$ & 369.77 & 11-15 & 374.92 & 16-7 & 358.59 \\
\hline 3-1 & 363.44 & 6-4 & 368.75 & $7-5$ & 369.05 & 9-1 & 369.94 & 12-13 & 371.92 & & \\
\hline 3-2 & 363.87 & 6-3 & 370.01 & $7-6$ & 371.24 & 9-12 & 348.42 & 12-14 & 342.45 & & \\
\hline 4-12 & 328.08 & 6-2 & 371.61 & 8-10 & 367.68 & 9-11 & 364.43 & 12-15 & 367.21 & & \\
\hline 4-1 & 359.20 & 6-1 & 371.84 & $8-9$ & 369.73 & 10-14 & 342.36 & 13-2 & 371.57 & & \\
\hline $4-2$ & 357.39 & 6-10 & 369.98 & 8-8 & 370.16 & 10-1 & 369.08 & 14-1 & 361.64 & & \\
\hline
\end{tabular}


Scheme 1: Substitution reaction starting from benzene and leading to toluene.

Scheme 2: Reaction pathway starting from toluene (1-1) and leading to 1-methylnaphthalene (2-1) (labeling see Figure 3).

Figure 1: Intrinsic (a) and modified (b) rate coefficients for the addition reaction of a methyl radical to benzene (see Scheme 1).

Figure 2: Modified rate coefficients $k^{\prime}(\mathrm{T})$ for the forward (in light grey) and reverse (in dark grey) reaction steps of the entire network (as defined in Scheme 2).

Figure 3: Singly methylated PAHs, methylation can occur at various positions as indicated (the methyl group is not displayed in the Figure).

Figure 4: Reactivity-enthalpy relationship for the hydrogen abstraction reactions at methylated PAHs by an attacking methyl radical.

Figure 5: Rate constants $\ln k(\mathrm{~T})\left(\mathrm{k}(\mathrm{T})\right.$ in $\mathrm{m}^{3} / \mathrm{mol} / \mathrm{s}$ ) as a function of $1 / \mathrm{T}$ (in $1 / \mathrm{K}$ ) for a random selection of five hydrogen abstraction reactions (numbering is conform Figure 3). The full lines correspond to abstractions at pure PAHs (leading to arylic radicals) whereas the dotted lines correspond to abstractions at methylated PAHs (leading to benzylic radicals). 

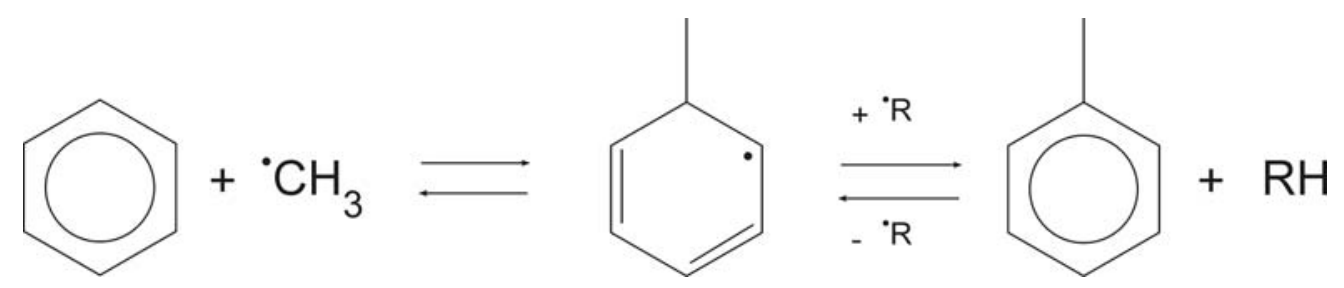

Scheme 1: Substitution reaction starting from benzene and leading to toluene. 
Scheme 2: Reaction pathway starting from toluene (1-1) and leading to 1-methylnaphthalene (2-1) (labeling see Figure 3 ). 


\section{Page 15 of 19}

1

2

3

4

5

6

7

8

9

10

11

12

13

14

15

16

17

18

19

20

21

22

23

24

25

26

27

28

29

30

31

32

33

34

35

36

37

38

39

40

41

42

43

44

45

46

47

48

49

50

51

52

53

54

55

56

57

58

59

60
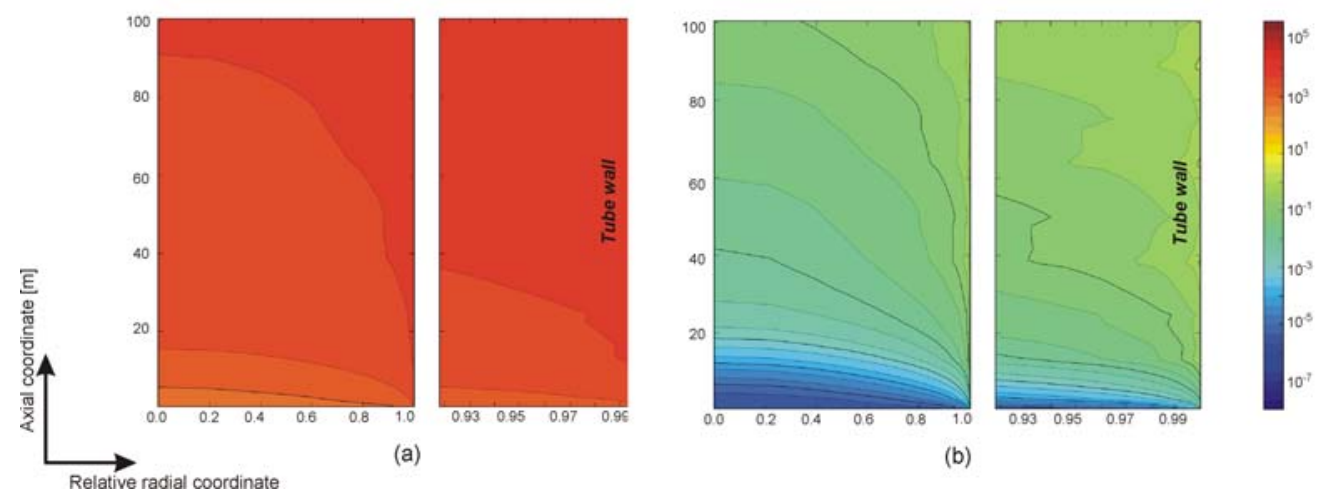

Figure 1: Intrinsic (a) and modified (b) rate coefficients for the addition reaction of a methyl radical to benzene (see Scheme 1). 


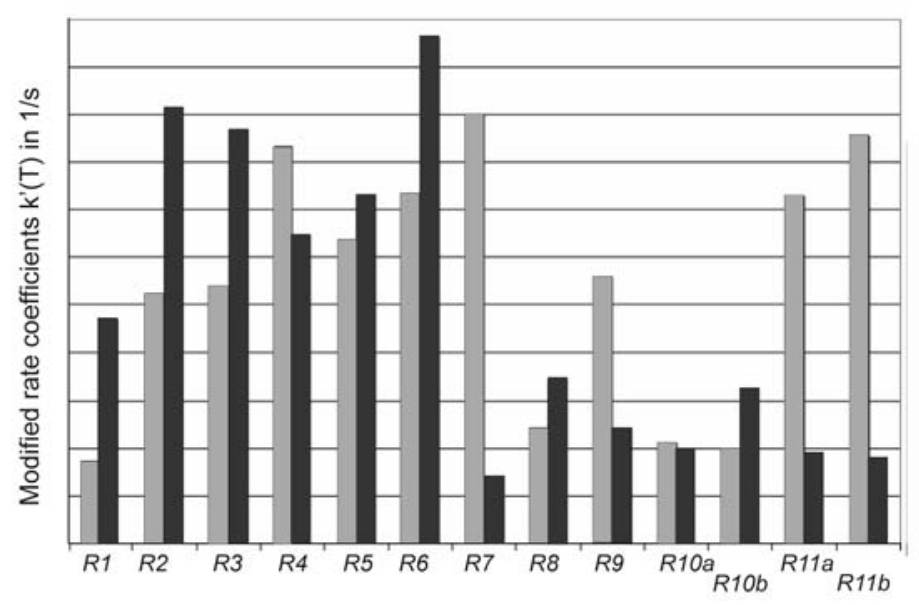

Figure 2: Modified rate coefficients $\mathrm{k}^{\prime}(\mathrm{T})$ for the forward (in light grey) and reverse (in dark grey) reaction steps of the entire network (as defined in Scheme 2 ). 
1<smiles>c1ccccc1</smiles>

2<smiles>C1=Cc2ccccc2[Te]=1</smiles>

3<smiles>C1=CC2=Cc3ccccc3SC2=IC1</smiles>

4<smiles>C1=Cc2cc3cc4ccccc4cc3pc2[Te][Tl]1</smiles>

5<smiles></smiles><smiles>C1=IC=Ic2ccccc2O1</smiles>

7

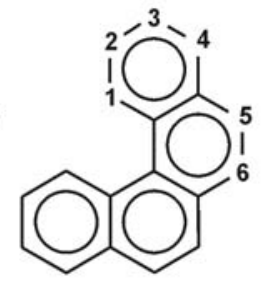

8

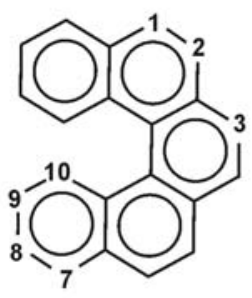

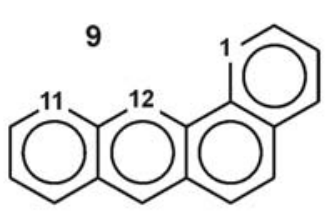

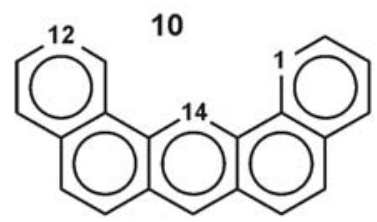<smiles>C1=C[Se]c2c3c(ccc2=C1)=Cc1ccc2ccc4c(c2c1[Se]3)[In]C=C4</smiles><smiles>c1c[se]c2c(c1)ccc1ccc3ccc4cc5ccc6cc[se]c6c5c4c3c12</smiles><smiles>c1cc2ccc3ccc4ccc5ccc6ccc1c1c2c3c4c5c61</smiles>

13<smiles></smiles>

15<smiles>c1cc2cccc3c4cccc5cccc(c(c1)c23)c54</smiles>

16

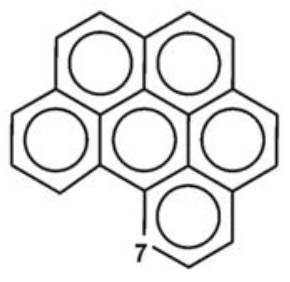

Figure 3: Singly methylated PAHs, methylation can occur at various positions as indicated (the methyl group is not displayed in the Figure). 
Figure 4: Reactivity-enthalpy relationship for the hydrogen abstraction reactions at methylated PAHs by an attacking methyl radical. 


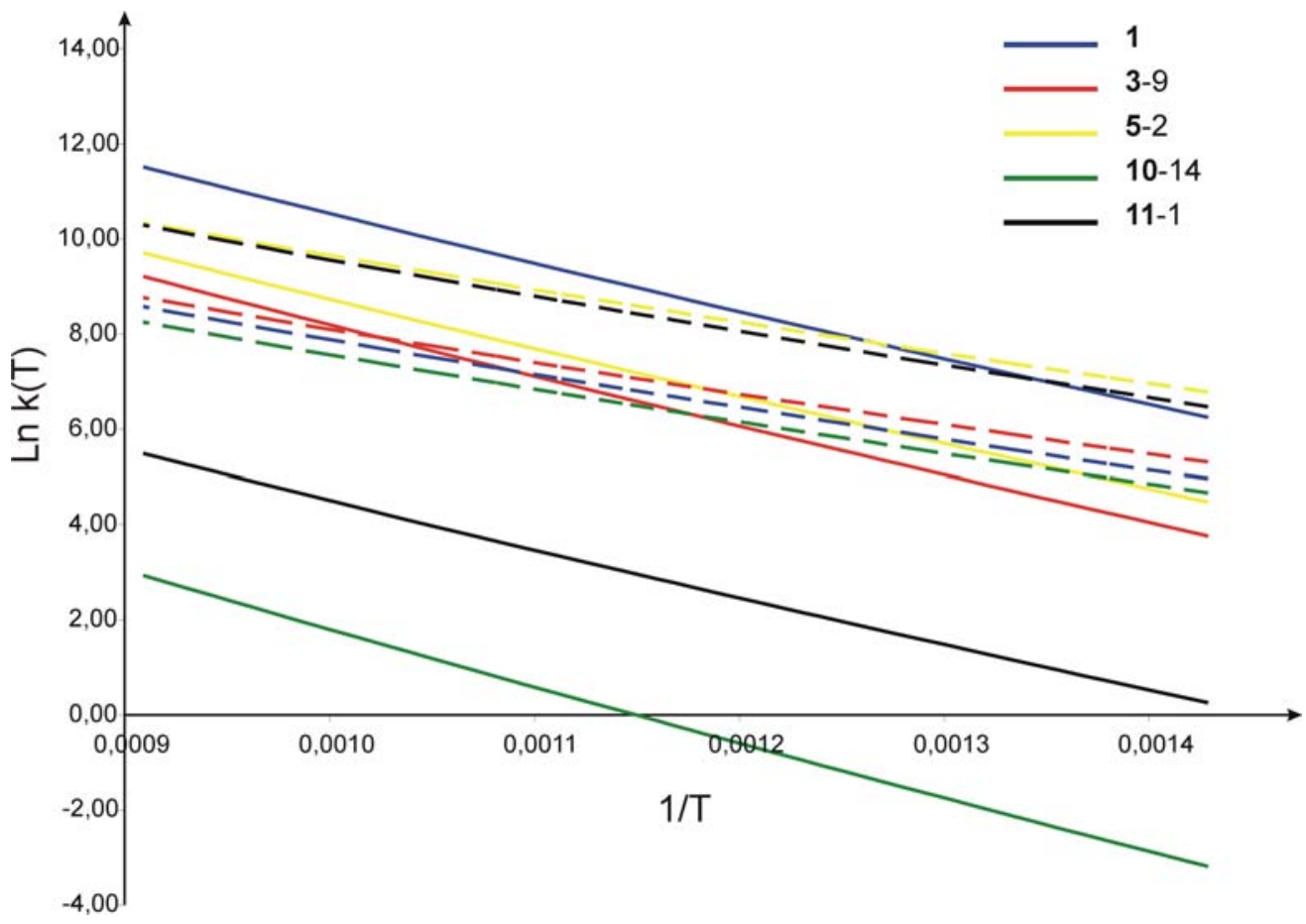

Figure 5: Rate constants $\ln k(T)(k(T)$ in $\mathrm{m} 3 / \mathrm{mol} / \mathrm{s}$ ) as a function of $1 / T$ (in $1 / K$ ) for a random selection of five hydrogen abstraction reactions (numbering is conform Figure 3 ). The full lines correspond to abstractions at pure PAHs (leading to arylic radicals) whereas the dotted lines correspond to abstractions at methylated PAHs (leading to benzylic radicals). 